\title{
On Location Learning: Authentic Applied Science with Networked Augmented Realities
}

\author{
Eric Rosenbaum, ${ }^{1,2}$ Eric Klopfer, ${ }^{1}$ and Judy Perry ${ }^{1}$
}

\begin{abstract}
The learning of science can be made more like the practice of science through authentic simulated experiences. We have created a networked handheld Augmented Reality environment that combines the authentic role-playing of Augmented Realities and the underlying models of Participatory Simulations. This game, known as Outbreak@ The Institute, is played across a university campus where players take on the roles of doctors, medical technicians, and public health experts to contain a disease outbreak. Players can interact with virtual characters and employ virtual diagnostic tests and medicines. They are challenged to identify the source and prevent the spread of an infectious disease that can spread among real and/or virtual characters according to an underlying model. In this paper, we report on data from three high school classes who played the game. We investigate students' perception of the authenticity of the game in terms of their personal embodiment in the game, their experience playing different roles, and their understanding of the dynamic model underlying the game.
\end{abstract}

KEY WORDS: augmented reality; participatory simulation; dynamic model; infectious disease; authenticity.

\section{INTRODUCTION}

Many experts in science education reform have called for the learning of science to be more like the practice of science (NSTA, 2003). These experts argue that incorporating the tools, intellectual processes, motivations, and consequences of science practice can enhance science learning. This model of learning draws upon a rich theoretical literature in constructivism, situativity theory, and communities of practice to create visions of authentic science learning for students. But what defines an activity as "authentic" is a source of some debate.

In this introduction, we discuss the debate about the nature of authentic experiences in the classroom

\footnotetext{
${ }^{1}$ MIT Teacher Education Program, 77 Massachusetts Ave. Room 7-337, Cambridge, MA, 02139, USA

${ }^{2}$ To whom correspondence should be addressed; E-mail: eric_r@ mit.edu
}

and address the role of technology in authentic learning. We describe two prior approaches to authentic learning technology-participatory simulations and augmented reality simulations - and explain how we have synthesized their features into a game which we argue creates a more authentic learning experience.

\section{What is Authenticity?}

The question of authenticity hinges on the context in which the task can be perceived as authentic. Lave and Wenger's (1991) vision of communities of practice centers on "real" problems taken on by participation in extended communities. In contrast, Practice Fields (Barab and Duffy, 2000) provide exploratory spaces where students participate in "reality lite" investigations with many or all of the consequences of real explorations removed.

Other theorists (Bereiter, 2002; Dede et al., 2005b) have called for classroom experiences to 
better reflect the complexity of 21 st century work and citizenship. A new set of skills is required from students in an information-based economy, and traditional classroom practices do poorly at teaching these skills. Students should be able to work with incomplete information, adapt to changing conditions, manage complexity, and fluidly create and share knowledge. These skills, which we may have formerly seen solely as a requisite for future professionals, are now demanded of many jobs and increasingly of every citizen. Every day people are faced with issues for which they must evaluate scientific evidence about claims such as the emergence of new diseases or global warming.

\section{Authenticity and Technology}

Technology can play an important role in integrating 21st century skills and mediating authentic experiences in the classroom (Roschelle et al., 2000) by facilitating the creation of communities of practice and incorporating many of the tools that practitioners use around issues of current scientific concern. Simulations provide the basis for one form of authentic learning by modeling specific aspects of real-world complex systems, allowing learners to experiment with the system either by manipulating parameters or participating inside the system and observing outcomes.

Simulations situated in rich, realistic 3D virtual worlds might be described as "heavily" virtual. These simulations are certainly engaging, but they depart so much from the actual world that they may feel less authentic. Learning environments that are "light" on virtual information, by contrast, provide less simulated sensory input, but remain closer to the actual world and can take advantage of its affordances for authenticity.

This balance between the strengths and weaknesses of virtual media in creating authentic learning environments has motivated us to explore a different take on technology-enabled authentic learning environments, namely through handheld computers instead of online environments. We use the affordances of handheld computers (e.g., portability) to "lightly" structure activities that take advantage of the authenticity of real-world environments and live social interactions with other participants. Through handheld-based simulations, students primarily interact with each other and with the real environment around them. This experience is augmented with information that is accessed periodically from their handheld computers.

The experience that we are trying to create may be seen in the light of Dewey's notion of "education through experience" (Dewey, 1938). While it may seem paradoxical to try to create real environments for problem solving through simulation, that is in fact what we are trying to do. Through interactions with the real-world context, natural social exchanges, and systems-based feedback, we attempt to create simulated experiences that reproduce crucial aspects of real-world experiences. The added advantage of such experiences is that they have direct applications outside of the classroom to real problems.

\section{Participatory Simulations}

Our first venture into handheld computer-mediated simulations was our Participatory Simulations. These simulations originally took place with the aid of wearable computers or Thinking Tags (Colella, 2000), and later through Palm computers (Klopfer et al., 2004). In both versions, each individual participant had a device through which they interacted with other players via short range infrared beaming. Perhaps the best known of these simulations (and most relevant here) is the Virus game. Players are co-located in a single classroom and have a goal of trying to meet as many other players as possible without getting sick. Players meet one another by beaming to each other's Palms (during which players' Palms are held about 48 inches apart); the Virus software tracks whom each player has met. After a while, some of the players start to get sick (shown by a happy face turning sad on their Palm and an alarm going off). The goal of the game quickly switches from the individual player's goal of meeting as many other players as possible without getting sick to a collective goal of figuring out things like from where the virus came, why some people got sick and not others, and what determined the timing of the people getting sick.

The Virus simulation has been used with thousands of students from elementary through graduate school. Colella (2001) found that these simulations engaged a broad range of students in problem solving and analysis of complex systems. Subsequent research on the handheld-based versions of these simulations (Klopfer et al., 2005) found that teachers were able to effectively engage students in a range of topics customized by the teachers ranging from biology (basics of epidemiology) to health (prevention of STDs) to history (transmission of the plague). Currently these 
simulations are a component of a biology curriculum under evaluation. Similar research has taken place using a different take on such Participatory Simulations (Wilensky and Stroup, 1999), which notably differs in that the participants in the simulation interact through a central onscreen representation instead of purely through distributed devices. This research also showed students readily engaging in discussion around complex systems.

The power of these Participatory Simulations comes from the underlying model. Each time the simulation is played, the game-play emerges slightly differently. While the model and parameters remain the same, the stochastic nature of events causes the situation to unfold in unique ways. This is important in understanding the nature of complex systems. Multiple replicates run on seemingly identical samples may behave differently due to slight differences in initial conditions or the timing and sequence of events. In this sense, these simulations provide authentic explorations of complex systems.

The underlying model in these simulations provides another sense of authenticity to the activity. Students can develop and test hypotheses by enacting "solutions" and observing outcomes. This provides a built-in form of authentic assessment. Students do not need to ask if they "got it right," but instead test solutions and measure outcomes that can help them evaluate their own understanding of the system. Whether they have mastered the concepts or still have gaps in their understanding becomes quite obvious.

What the Participatory Simulations lack, however, is a sense of authenticity in terms of grounding the experiences in real situations with a rich context and meaningful consequences. The simulations are purposely quite abstract and generic. For example, in the Virus game, no specific disease is named, affording teachers the flexibility to use it to discuss a variety of diseases. The normal consequences of being sick are also absent from the game. While students are apt to think of themselves as sick (and are often heard saying, "Oh, I got sick!"), being sick does not affect their ability to take actions in the game. They also do not have any tools such as diagnostic tests and medicines for dealing with people who are sick.

\section{Augmented Reality Simulations}

Another take on the use of handheld simulations is our Augmented Reality (AR) simulations (Klopfer and Squire, 2006; Squire and Klopfer, 2006). In these simulations, players use location-aware handheld computers to participate in large scale complex investigations that use simulated information on their handheld computers combined with the physical realworld environment and context in which they are located. The first of these simulations, Environmental Detectives, placed students in the role of environmental engineers trying to uncover the source of a toxin that had leaked into the groundwater. The students (upper high school and university) investigate this scenario in the actual geographic location that they are investigating (i.e., if the scenario takes place on a high school campus, students need to walk around that actual campus as a part of the game). While Environmental Detectives was originally developed for use on a university campus, it was relocated and reauthored for various high school campuses and nature centers. The students needed to take into account many of the real constraints of that particular location: use of the land and water, nearby water sources, topology, attitudes of the local community, visibility of potential remediation, and use of chemicals in the vicinity. Some of this information was directly incorporated into the digital content of the game itself. Specifically, students' handhelds provided them with supporting documents, interviews with on-location experts and witnesses, and virtual samples that they could take at their current location. However, students also collected additional relevant information by walking around and observing the real-world environment. The design goal of these simulations was to help students negotiate between many types of data that would normally be encountered in this field of practice, including primary and secondary data as well as realtime, live data and stored, digital data.

We have previously argued (Klopfer and Squire, 2006; Squire and Klopfer, 2006) that these augmented realities provide yet a different sense of authenticity between communities of practice and practice fields. They involve many of the constraints of real investigations (time, resources, etc.) as well as the real possibility of failure and were developed in consultation with environmental engineers and scientists to match the real intellectual challenges of their jobs. But these simulations also provide the real tools, techniques and approaches of science practice. Furthermore, they are grounded in realistic scenarios based on the possible, if not the actual.

What the AR simulations to date are missing is a built-in system of feedback. The simulations are based on real locations, real scientific data, and real practices; but they do not incorporate dynamic models. 
The data that students collect is pre-generated offline and built into the games. This means that they cannot build and test solutions inside of the game, as one can do in the Participatory Simulations. As a result, we have used alternative forms of assessment, such as having the students present their evidence before a jury. However, this removes one of the senses of authenticity, that the only way to know the "truth" is to design and test solutions, and instead conveys the sense that someone outside of the system is an omniscient expert with the answer. This can be mitigated using clever teaching, but detracts from the authenticity of the entire experience.

\section{"Participatory Reality"}

The ideal educational tool would incorporate the authentic experience of on-location learning, including the tools, intellectual traditions and community aspects of AR, coupled with the ability to interact with an underlying model. We might think of such a system as a "Participatory Reality"-in which students participate in the system and affect its outcome, yet the simulation remains grounded in reality through real spaces, contexts, and locations.

Drawing upon advances in locative technologies (namely using Wi-Fi positioning) and client-server communication (handhelds that can connect wirelessly to networks across large spaces), we designed and implemented a new generation of AR that provided such a "Participatory Reality". This game, Outbreak@ The Institute, simulates a disease outbreak on a university campus and challenges players to contain it using tools such as diagnostic tests, vaccines and quarantining.

\section{RESEARCH QUESTIONS}

In our study of an Augmented Reality game, we seek to answer two research questions. First, do students perceive the game as an authentic experience? We expect that the authenticity of the game will manifest in some ways similar to those seen with the earlier augmented reality games, which were also location based and involved collaborative work on realistic problems. Additionally, we hypothesize that the dynamic aspect of the game may contribute to the authenticity of the game in powerful ways. These include a sense of personal involvement and the realistic enactment of professional roles.

Our second research question is, do students understand the dynamic nature of the model under- lying the game? The disease outbreak modeled in the game is a complex system, in which the players are participants. We expect their direct, personal involvement in it to contribute to their understanding of it on a system level (i.e., the way diseases spread through the population).

\section{GAME OVERVIEW}

\section{Scenario}

Players of Outbreak@The Institute participate in a fictional scenario: the outbreak of an emerging disease. Specifically, they are confronted with an outbreak on a university campus of a new form of Avian Influenza, or Bird Flu, which is very dangerous because (in this fictional scenario) it has become transmissible between humans. Several students have come to campus from around the world for an international robotics competition, and some are already exhibiting flu-like symptoms. Players may encounter both Bird Flu and the common Seasonal Flu, which have very different outcomes but can be difficult to distinguish in the early stages. The players must work together as a team of diverse roles to gather information and use the tools available to them to contain or stop the outbreak as best they can.

This particular scenario involving bird flu was chosen for several reasons. First, it is an issue of great public interest and concern. We are bombarded by images and articles on the spread of bird flu between birds and people, as the media plays on people's fear of a global pandemic. Interpreting this information can be a challenging task. This activity builds on that interest and hopefully provides valuable skills in making more sense of current and future dilemmas. Second, previous research (Colella, 2000; Klopfer et al., 2004; Wilensky and Stroup, 1999) has demonstrated that students readily engage in inquiry around dynamic models of disease. And finally, the community of epidemiologists and public health experts, recognizing the importance of the general citizenry to be better informed about emerging diseases, provided valuable input and feedback in the design of the game with respect to models, content, and practices.

\section{Technology}

Outbreak@The Institute is played on handheld computers, commonly referred to as personal digital assistants (PDAs). Each player has a PDA with 
which she can observe her status in the game and take actions. The PDAs are connected wirelessly to a network, which provides the PDAs (and the players) with positioning information and connects players to each other as well as game content through clientserver connectivity. There are three key components to the technology that enable the core functionality in this game. These are Location Basis, the Disease Model, and Player Status.

\section{Location Basis}

The PDA connects wirelessly to a server, which tracks in which building the player is currently located (based on Wi-Fi signal strength). This allows the game server to deliver information based on the player's real-world location. As players move from building to building, different virtual characters appear on the screen of their PDA. Players can then take actions such as interviewing the virtual characters to get textual clues or treating them using medicine. The server tracks the status of all players, allowing them to interact in a single virtual world, so that the actions of one player can affect all the others.

\section{Disease Model}

An underlying probabilistic model of disease transmission is used to create realistic patterns of infection. Each player and virtual character has an antigen count, representing the number of virus particles (or titer) in their body. This quantity is not directly visible to players in the game; but, as the quantity increases, the player's health level (which is visible) drops. Seasonal Flu and Bird Flu have different equations governing how their antigen counts change over time. Players and virtual characters can infect each other, with a probability dependent on their antigen counts and the amount of time they spend together in a room.

\section{Player Status}

A player's status includes her current health, shown by a meter, which decreases if she becomes infected with a virtual disease, and her inventory of items picked up during the game. The player's health is affected by her disease state (modeling which, if any, disease she has and how far it has progressed), time, and medicines. Game items, such as diagnostic testing kits and vaccines, are scattered around the virtual landscape, as well as being in the initial pos- session of some players. These items provide specific functionality and may be restricted in use by role (see below).

\section{Roles}

Players join the game in one of three possible roles, each of which has different abilities in the game. With small numbers of students in each class, we wanted to enable enough diversity in roles to encourage jigsawing of complementary information, but also to provide some redundancy where information may be missed. The three roles that we chose were as follows:

Medical Doctors can use the various types of medicine in the game to treat players and virtual characters. The medicines include palliatives (which reduce symptoms only), vaccines (which prevent infections), and cures (which stop the course of disease).

Field Technicians can diagnose diseases. First, they use a sampling device to take a blood or mucus sample from another player or virtual character. They put the resulting sample into an analyzer, which reports the presence or absence of a disease. False negative readings are possible due to a threshold for antigen count before it can be detected and due to error built into the analyzer, representing its inaccuracy.

Public Health Officials can quarantine virtual characters. There is a special location in the game representing a quarantine room, in which diseases are not transmissible. The Public Health Officials can use a special item that allows them to transport a virtual character to the quarantine room.

\section{End Game}

Players are not given specific criteria for "winning" the game. Instead, they have only the loosely defined tasks of gathering information and containing or stopping the outbreak, and a limited total amount of time to play. As a result, players must decide for themselves what their goals should be throughout the game. Allowing the students to define goal states, rather than specifying them, creates a more realistic situation in which they must evaluate trade-offs and decide on a satisfactory balance. In the group meeting at the end of the game, students discussed the information gathered (for example, scientific knowledge about bird flu, and hypotheses about which virtual characters were initially infected) and estimated their degree of success in containing the outbreak based on their observations of which real and 
virtual characters got sick. The lack of specific criteria for winning makes the game more like real-world problem solving tasks, which are often ill defined.

\section{Game Walk-Through}

The following is a descriptive account of a common sequence of events that a player might experience when playing Outbreak@ the Institute:

Eric arrives along with his classmates to play Outbreak@ the Institute, and they all watch a brief presentation introducing the game. Eric is assigned to partner with his friend, Judy, and the pair is given the role of Field Technician. They receive one handheld computer to share between them. Judy initially takes the device and logs in. The game starts up with the screen displaying an overview of their status. At the top they see their health meter, which starts at $100 \%$. They also see the name of the current room they are in: the hospital. The students are physically located in a classroom at the center of campus, but in the game this classroom is designated the hospital, an impromptu triage center. The hospital room contains some important virtual items that also show up in a list on this screen, such as a mucus swab (for taking mucus samples, to test for the seasonal flu), some Tylenol, and the "accu-flu analyzer." As other pairs of students log on, their names appear on the screen as well, indicating that they are currently in the hospital room. There are also some other people listed in this room, with unfamiliar names; these are virtual characters. Judy selects one of these virtual characters, whose name is Larry Lynch, and presses a button to "interview" him. A window pops up showing the text of his response, which includes that he is the pharmacist, and that medications exist for preventing and treating the flu, but these are only available to medical doctors (which Judy and Eric are not; their role is Field Technician).

Before leaving the room to explore other locations in the game, several pairs of students seem interested in poking around with the software to see what they can do. Eric suggests that he and Judy try out testing someone for a disease, since they are a field technician and that is their special ability in the game. First they need to take a sample. They find in their inventory screen that they already possess a mucus swab, which will allow them to take a mucus sample. They click a button to use it, select their classmate Trey (playing a medical doctor), and a moment later a mucus sample appears in their inventory. Next, they must analyze the mucus sample. They can choose between the "rapid-flu analyzer," which they can carry around with them, or the "accu-flu analyzer," which takes a little longer to analyze the sample, and has to stay in the hospital room, but which is more reliably accurate.
They select the accu-flu, and send the mucus sample to it. After a short delay, they receive a "measurement" back, a little report, which informs them that Trey does not have the flu. At this point a team that includes two other pairs of students is gathering to leave the room. They hope to find additional virtual characters in another room on campus, who can hopefully lead them to the person suspected of being infected with bird flu. The team currently has a doctor and a public health official, so Eric and Judy join them and head off.

The team of three pairs works its way across campus through a few rooms, and interviews a few different virtual characters. In the biology building, they find Ariel Cohen, an influenza researcher. She gives them some details about the different strains of flu, and how immunity develops. In another building they meet Michael Somer, who is organizing an international robotics competition. Michael doesn't say anything specific either to Eric and Judy's role. But they compare notes with Maya and Oren, who are playing the public health official role, and learn that they got a different response from the virtual character Michael: since they're a public health official, he told them that some of the international students just arriving for the competition have come from the countries where bird flu is known to be spreading. Judy decides that they should test Michael for the bird flu, which they do by taking a blood sample, and he turns out to be healthy.

Later in the game, after traveling to several other locations such as the student center and the fitness center and interviewing several virtual characters in each place, Eric and Judy take a break in the computer science building, where they have just interviewed Tami Meiji, one of the students involved in the robotics competition. The medical doctor and public health official teams have joined up with them. Suddenly, Eric notices that their health meter has dropped a tiny bit below $100 \%$. He calls out to his teammates that he's sick. Immediately they begin to edge away, but he asks for medicine. Fortunately the medical doctor team has some anti-viral medication in their inventory. Before administering it, they decide to put on virtual items, gloves and a mask, which should decrease the chance they will also get infected. Nonetheless, soon after giving out the medicine the teams decide to leave for another room, hoping to track down more infected people and treat them.

Eric and Judy's health level continues to drop rapidly. They call the doctor team on their walkie-talkie, but get no response. As they wait anxiously, Judy realizes that it must have been Tami Meiji who infected them: the medical doctor team had noticed that when they interviewed Tami, she said she was not feeling well. They had decided to give her some Tylenol. But does Tami have the seasonal flu or the bird flu? The public health official team wants to quarantine Tami right away, to protect 
them all. But Judy wants to find out which disease she has! She insists that they stay with Tami long enough to run both a blood test and a mucus test to find out for sure...

This walk-through highlights several important aspects of the game. Collaboration is shown resulting from the distinct abilities of different roles in the game, and the fact that virtual characters respond to each role differently. Players are shown making inferences that combine multiple sources of information (testimony from virtual characters, and results from imperfect diagnostic tests). It also illustrates the urgency and emotional response that players experience when they become infected or encounter sick people during the game.

\section{RESEARCH METHODS}

\section{Participants}

The participants in the study were three small classes, totaling 21 students (15 girls and 6 boys), from an urban mathematics and science public high school. The students were high school seniors who participated in the activity as part of their class on medical technology. The school's population is $46 \%$ African American, 23\% Asian, 20\% Hispanic, and $11 \%$ White. Sixty-two percent of the school population is classified as "low-income."

\section{Procedures}

The week prior to the study, researchers visited each of the participating classes, giving the students a presentation on the game (an overview of the scenario and the abilities of each role) and administering the presurvey. The next week, each of the three classes came on a field trip on a different day to play the game. On each occasion, students were first given another presentation on the game, this time with additional details about the capabilities of each role and instruction on how to operate the PDAs. The students were put into pairs, with each pair using one PDA and acting as one role in the game. Each pair carried a walkie-talkie set to an open channel for communicating over a limited distance with the other players. Six-person teams were formed by combining three pairs, with one pair of each role (a medical doctor pair, a field technician pair, and a public health officer pair). They then played the game for approximately $2 \mathrm{~h}$. At the end of the two hours the students gathered for a discussion, and were given the post-survey.
One of the assessment components involved a diagramming task, to ascertain student understanding of the dynamics of disease systems and how individuals influenced and were affected by the spread of disease. To prepare students for this diagramming task on the pre- and post-surveys, students were given a special training exercise (described below) to introduce them to the concept of an "influence diagram." An influence diagram is a drawing with boxes representing quantities in the world, and arrows connecting the boxes showing that the level of these quantities affects one another (this is a simplified form of the causal loop diagram (Senge, 2000)).

The pre-game classroom visit included an activity in which students constructed an influence diagram as a class. First, students were given a printout of a classic email forward hoax. A discussion was opened by asking them if they believed what the email said to be true and why. Next, the researchers began drawing an influence diagram on the board, showing some factors that might influence the number of people who believe the email to be true (see Figure 1). These factors included, for example, the email's believability, its use of realistic details, people's level of skepticism, and the number of people who actually forwarded it. Students' own ideas for factors were elicited and added. Then, arrows were added to the diagram, showing how each factor influenced the others. For example, the number of people who believe the email affects the number of people who forward it, so an arrow in that direction was drawn. Also, the number of people who forward the email affects the number of people who believe it, so that reciprocal arrow was

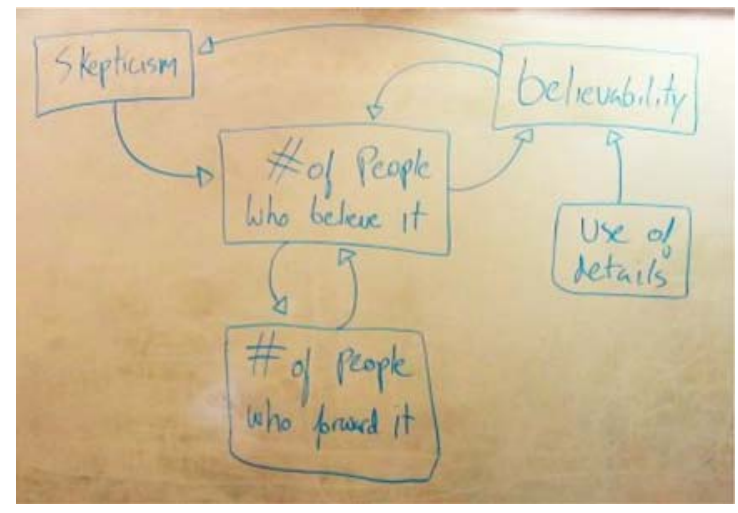

Fig. 1. Sample of an influence diagram constructed in class. The diagram shows factors influencing the number of people who believe an email forward hoax. 
drawn as well. Following this exercise, students were given the pre-survey.

\section{Instruments}

\section{Pre- and Post-Surveys}

Surveys were administered before and after the game. The survey consisted of two parts: (1) questions on students' goals in the game, and (2) an influence diagramming task.

The goals questions were designed to get at students' own views of their goals in the game and their success in achieving them. They included a question in which students ranked a list of possible goals in order of importance. This question was identical on the pre- and post-surveys.

The influence diagramming task asked students to list at least five factors in the game that would influence the number of sick people. Then they were asked to draw a diagram, started for them with a box labeled "Number of sick people," and to include each of their five factors in boxes and arrows between them to show how they influence each other. This task was presented in an identical way on the pre- and post-surveys.

\section{Game-Play Video}

Each group of players was videotaped as they played. Selections from the video were transcribed to look for patterns in play and conversation.

\section{Interviews}

Brief interviews were conducted with a random subset of students from each class after they completed their post-survey. Students were asked to compare their pre- and post-survey responses and diagrams and to reflect on any differences between them. The interviews were videotaped and transcribed.

\section{FINDINGS}

\section{Authenticity of Personal Involvement}

\section{Personal Embodiment in the Game}

Students behaved during the game in ways suggesting that they felt personally embodied in the game. By "personal embodiment" we refer to the feeling that one's body is physically interacting with the virtual environment. For example, when the bar graph on the PDA screen representing the students' health started to drop, they spoke and acted as though they were actually sick. When they were not sick, they responded to sick players with fear and alarm, attempting to physically move away from them. These responses indicate that students experienced the threat of disease in the game in an intuitive and visceral way, as though it were real.

In the game-play video, one pair of students (students 2 and 3, below) can be seen edging away from another pair they suspect to be sick:

Facilitator: How are your health meters?

Student 1: um, we're s... well it's starting to drop.

Student 2: Let's get away from them.

Student 3: They're sick! [points at student 1 and her partner] Put on a gas mask!

Student 1: It's like, just a little bit...

Student 1 starts to say that she is sick, but switches to simply saying that her health meter is dropping; later she emphasizes defensively that it has only dropped a little bit. Meanwhile students 2 and 3 react with exaggerated fear. Student one's reluctance to admit she is sick reflects the seriousness with which she treats being sick, and the importance of her classmates' reactions to her health status.

In a post-game interview, one student described the influence of his fear of getting sick on the course of action that he took:

\footnotetext{
Student: On my pre-survey [I gave a high ranking to the goal] "keep everyone from getting sick." But when the game really started, I just wanted to find out who was sick, so I could stay away from them.

Interviewer: That's interesting. So what experience made you change that?
}

Student: When I realized I could get bird flu.

These examples show how students' speech and physical movements reflected their experience of the disease in the game as an authentic danger. This experience affected both their social interactions and their actions within the game. 


\section{Shift Toward Person-level Goals}

Perhaps reflecting their personal embodiment in the game, students' perceptions of their goals changed during the course of the game, from knowledge-based goals to more personal and team-based goals. On both the pre-survey and the post-survey, students were asked to rank a list of seven goals in order of importance. The list that they had to choose from was as follows:

- Keep myself from getting sick

- Keep my team from getting sick

- Keep everyone from getting sick

- Find out who was sick first

- Carry out an effective strategy

- Learn about strategies for responding to a disease outbreak

- Learn about bird flu

As shown in Figure 2, before playing the game, students' highest rankings were for "learn about bird flu" and "learn about strategies for responding to a disease outbreak," reflecting a distant, impersonal view of the game, and goals that one might be accustomed to seeing in school-based learning activities. After the game, these two items received the lowest rankings. The highest rankings on the post-survey were for "keep myself from getting sick" and "keep my team from getting sick." There was a significant difference between rankings on the pre- and post-surveys for "learn about strategies for responding to a disease outbreak" ( $t$-test, corrected for multiple comparisons; $p<0.007)$. The difference was marginally significant for "learn about bird flu" $(p<0.009)$.

The change in rankings overall reflects a shift away from meta-game goals, such as learning about bird flu, and towards individual goals relevant to game-play, such as staying healthy. It is relevant to note that there were no negative consequences to getting sick in this game, other than those imposed by the players themselves, and the possibility of infecting others (players cannot "die" in the game).

Post-game interviews showed that students were aware of their shift in goals. The interviewer asked students to compare their rankings of goals on the pre- and post-surveys. Students were able to articulate that their goals had shifted generally towards keeping themselves and their team from getting sick. This interview after the activity shows one example: Interviewer: So are there big differences in your
goal rankings?

Student: Yeah... Everything basically changed... Everything that was in the beginning on the presurvey went towards the end on the post.

Interviewer: So what are some of those things?

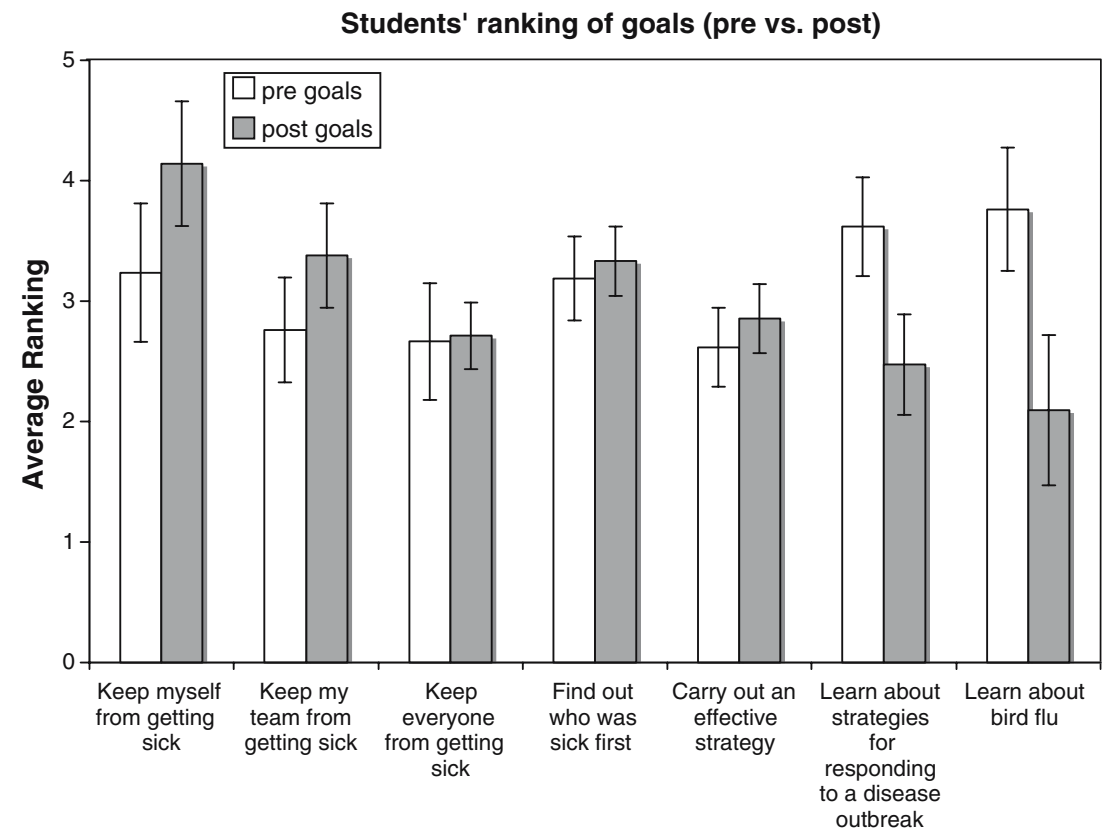

Fig. 2. Students' ranked importance of goals in the disease outbreak game, before and after playing (error bars represent one standard error; $n=21$ ). 
Student: ... On the pre-survey, keeping myself from getting sick and like other people from getting sick was like at the end of the list, and then they're at the beginning after I played the game.

Another student, giving reasons for this shift in goals, described the importance of keeping herself from getting sick:

\begin{abstract}
Interviewer: So what experience made you change your ranking [moving "keep my team from getting sick" to a higher ranking on the post-test]?
\end{abstract}

Student: I think it was because when we were all together, it was kinda like, you're getting sick, oh no, I have to help you, and then also, like, wait, you're getting me sick, too! So, y'know, that part of it became an important thing.

In post-surveys, students did not give their highest rankings to "keep everyone from getting sick," even though this would be an appropriate goal given the structure of the game. Instead, they seem to have been focused more locally on themselves and their teammates. As we saw above in the evidence on students' personal embodiment in the game, these responses indicate the importance of the personal impact of the simulated disease. This impact motivated students' actions in the game: they worked to contain the disease to protect themselves and their classmates, not because they were performing an impersonal task or experiment. The intrinsic value of goals is a powerful benefit of authenticity: students are motivated to learn because they have a personal interest in the outcome. The potential trade-off is that the game might become "too real," and instead distract them from the work of learning.

In the next section we'll see that the process of learning is also influenced by the authenticity conveyed by the practices associated with the three roles in the game.

\section{Authenticity of Practices in the Game}

\section{Authentic Roles}

The division of abilities in the game into three distinct roles felt authentic to some students. The students were able to understand what tasks were associated with their own roles, and which tasks were associated with their teammates in different roles. The tasks that students were responsible for became tightly associated with them, and each student needed to take responsibility for those tasks or there would be individual and group consequences. One student simply said in an interview, "I had a lot of fun. I thought I was a real doctor."

Another student adopted the habits of mind of a medical doctor in such a situation who might feel the obligation to cure all of the patients. She said that after the game ended, "I felt like, there was still probably someone else out there, somebody that really had the bird flu that we really didn't get to..." This suggests that the student felt some attachment to the virtual characters and a sense of responsibility to treat them.

In the transcript below from the game-play video, two students discuss whether or not a virtual character is sick. Student 2 is playing the doctor role, and considering whether or not to give some medicine to the virtual character. He's relying on information from a third student, playing the field technician role, to give him back some test results.

\section{Student 1 (Public Health Official): Nobody's sick! \\ Student 2 (Medical Doctor): Yeah but we talked to the other dude. He's having a party... They're doing a mucus test on him, to see if he's sick or not. Hopefully he's not sick.}

\section{Student 1: What do you mean, hopefully he's not sick? Then you're out of a job!}

This interaction suggests the extent to which students saw their abilities within the game as tied to their roles in authentic way, like a real occupation.

\section{Importance of Communication and Collaboration}

The three roles were also effective in fostering collaboration through jigsawing. Each of the roles was dependent on the others both for information and for action. For example, doctors needed to rely on technicians for taking samples, which they could in turn act on by providing medicine. Students grasped the resulting importance of communication and collaboration for success in the game. One student explained in an interview how the teams separated too much and so couldn't share information (for example, about which virtual characters were known or suspected to be sick):

\footnotetext{
"Because we didn't know how serious it could be or how fast it could spread, we decided to split up. And that obviously wasn't a good idea, because we lost communication..."
}

The same student shows an understanding of the interdependence of the roles, as well, pointing out 
that the group that did stay together did not have one of each role and so didn't have all the necessary abilities:

\begin{abstract}
"...also we spread out too much, but at the same time we didn't spread out enough because some people stayed together, but the way we stayed together was not right, because we had the public health officials and the field techs together but we should have really kept the doctors close by too."
\end{abstract}

There is a tension built into the game between staying together as a large group, and splitting up. Staying together allows the group to collaborate effectively, but the game is spread out over a large physical area, so simply to visit all the locations and gather enough information they need to split up to some extent. There is also a danger of being in a group, which is that if one player becomes infected they could all become infected. This student recognized some of this tension in the game and the complication it adds to the effort to collaborate.

Another student, explaining her influence diagram, noticed that her pre-game diagram did not include communication, but the post-game diagram did:

Interviewer: [What was] the difference between this diagram and the one you did before?

Student: I don't think I had anything about communication... I didn't realize how important it was for us to talk to each other until we actually did this.

The importance of communication and collaboration in the game mirrors their importance in realworld problem solving (as in, for example, a real disease outbreak), lending authenticity to this aspect of the game.

\section{Authenticity of the Game World}

\section{Game World as Dynamic System}

Some students understood that the augmented reality world of the game was dynamic, changing over time in response to their actions. The game includes a disease model, which specifies rules for disease transmission, the resulting change over time in the health of an infected person, and the effects of medicines.

One system-level consequence of this set of rules is that the disease can spread exponentially: a small number of infected people can in turn infect a much larger number of people, and the rate of new infections increases rapidly. This student displayed an under- standing of this aspect of the game in a post-game interview:

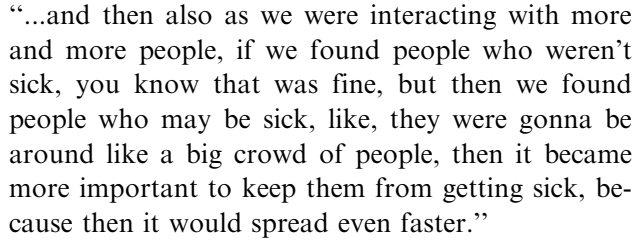

"...and then also as we were interacting with more and more people, if we found people who weren't sick, you know that was fine, but then we found people who may be sick, like, they were gonna be around like a big crowd of people, then it became more important to keep them from getting sick, because then it would spread even faster."

More generally, because of the dynamic nature of the model underlying the game, events unfold according to a complex network of causes and effects. Students' influence diagrams reflected an understanding of this complex causality, with the diagrams on the post-survey showing more interconnections between factors. Students drew significantly more arrows on post- than pre-diagrams $(p<0.02)$. The median number of arrows increased from 5 to 7 . This change indicates an increased understanding of the activity as a complex system in which each factor influences and is influenced by many others. We did not analyze the diagrams for accuracy, due to the subjective nature of the diagramming task; the increased connectivity on the diagrams, independent of their accuracy as representations of the complex system in the game, is sufficient to show that students gained an increased appreciation of its complexity.

Figure 3 shows the pre- and post-diagrams of a student who showed both an initial understanding of the diagramming task, and a shift toward a more sophisticated understanding of the game world. Her pre-survey diagram shows an understanding of both the nature of the game and of the diagramming task. The diagram is well formed, with a reasonable set of factors and arrows connecting them. For example, the number of people given medicine is influenced both by the cause of infection and by the number of people who don't say they are sick. This in turn affects both the number of people quarantined and the number of sick people.

This student's post-diagram shows a more sophisticated understanding of the dynamic game world, with better factors and additional arrows. The factors now distinguish between people exposed, people infected, and people who are sick, suggesting a more sophisticated mental model of disease. The factors also include sampling methods (used to diagnose and distinguish between illnesses in the game), which are a crucial part of the complex causality in the system. Interestingly, the student drew a direct connection between sampling and the number 

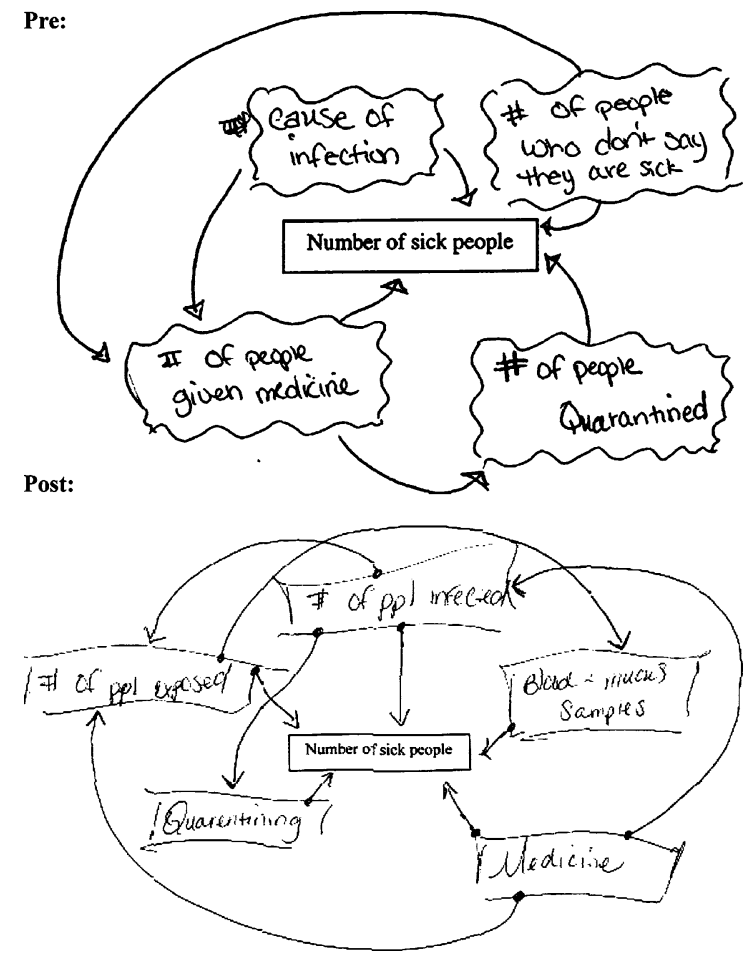

Fig. 3. A student's influence diagram on the pre- and post-survey. They show the factors the student thought influenced the number of sick people in the game, and arrows connecting them to show how they influence each other.

of sick people. In general, sampling does not directly affect the number of sick people. Sampling does however play an important indirect role: accurate diagnosis allows more sick people to be treated or quarantined, reducing the total number of sick people. Of course, a player taking samples without wearing protective gear (gloves and masks, in the game) could himself become infected, so there is an argument for the direct link as well.

In this student's post-diagram, the number of arrows increases from 7 to 10 , suggesting a more nuanced view of causes and effects in the game. For example, the student indicates that the number of people infected influences the number of people exposed, quarantining (presumably, the number of people quarantined) and the number of sick people.

Interestingly, there are no feedback loops in this diagram. It shows the number of people infected influencing the number of sick people; arguably, the number of sick people in turn has an effect on the number of people who get infected. Some students included this type of loop in their diagrams.

\section{Misconceptions About the Disease Model}

Students may have had some misconceptions about the disease model underlying the game. The game models infection as a discrete event, but students appeared to treat the presence of sick people as having a continuous effect.

In the game, as in real life, when you spend time near someone with the flu, there is some probability that you will become infected. At any given time, you either are or are not infected. Once you do become infected, the flu virus multiplies in your body, and you gradually become sick. In the game, your health meter gradually begins to drop, and at that point it does not matter where you are or whom you are with, because the infection is progressing all by itself. The only things that can affect your health in the game at that point are curative or palliative drugs.

Students appeared to understand disease transmission instead as a continuous effect, where the harm to their health accumulated over time spent in the presence of a sick person. This is a bit like the real effect of radiation poisoning: the longer you are exposed to a constant source of radiation, the more damage accumulates to your tissues, and the greater the detrimental effect on your health.

One student, for example, after noticing her health meter beginning to drop, said this: "uh, our health dropped again. I think we need to leave this room. Let's leave this room. Let's go quarantine ourselves." She is implying that by leaving the room, she can stop her health meter from continuing to drop. She can't; the only way to do that is by getting treated with medicines.

The misconception may be caused by a lack of knowledge about the underlying mechanism of disease transmission. In the pre-game presentation, students were told the rule that the more time they spend in a room with a player or virtual character who is sick, the more likely it is they will be infected. They may have interpreted this simply as "spending time with sick people is bad for you," and thus conclude that the more time you spend with them, the greater the effect on your health. Students may benefit from a more detailed description of the model of infection as a discrete event that initiates a deterministic disease time-course.

A related misconception appears around vaccination. Some students seemed to think that vaccination could be helpful even after the initial infection event. For example, one student said: "I think I could give you guys some flu vaccine, in case you guys are 
sick." In the game, vaccination is only helpful to prevent an initial infection; once you are infected, a vaccine will have no effect (it's worth noting that this is indeed the case for the real-world flu, though it may not be for other diseases). Students may have seen vaccination as a protection against the continuous harmful effect of disease transmitted by proximity to a sick person, rather than a protection against a discrete infection event.

\section{DISCUSSION}

\section{Authenticity}

Our first research question asks whether students perceive the game as authentic. The findings suggest that they did in several ways. Students felt personally embodied in the game, as shown by their verbal and physical reactions to the virtual disease in the game. Their shift on the survey from meta-level to personlevel goals reflects this embodiment. Students also experienced their roles in the game in authentic ways. During interviews and game-play, students demonstrated the seriousness and responsibility with which they treated their roles. They also showed an understanding of the interdependence of the roles and the importance of communication in the game. Finally, students appreciated the dynamic nature of the game world, seeing that their actions had an effect on the outcome of the game in a realistic way. Together, these findings suggest that the game created an authentic experience for the students, meeting many of the design criteria for practice fields (Barab and Duffy, 2000), while adding the affordances of a dynamic underlying model.

The location basis of the game may have contributed to authenticity in a different way than it did in our earlier augmented reality games. Here we find it useful to draw a distinction between "immersion" and "personal embodiment." By immersion we refer to sensory experiences that contribute to a sense of place. By personal embodiment we mean a feeling that one's body is physically interacting with the simulated environment. In the earlier augmented reality games, such as Environmental Detectives (Klopfer and Squire, 2006), players draw contextual information from their physical surroundings (such as the arrangement of buildings, green space, and rivers) and use this to reason about the problem presented. This contextual information contributes to immersion. In Outbreak @ the Institute, players could, for example, go to the campus medical center to find a virtual doctor, or to the student center to meet a virtual undergraduate. But in some cases, such as the quarantine room, which was represented by a central lobby space, there was no connection between the physical surround and the virtual location. Where real locations have contextual cues connecting them to their virtual identities, becoming immersed in the game world may be easier. Our observations suggest that what was even more important in the case of Outbreak@ the Institute was students'personal embodiment in the game, which was afforded by the location-basis of the game, but not necessarily through specific location-based contextual cues. The ability to move around in groups across a large campus, interact face-to-face, and think and act physically and spatially (for example, hurrying to leave a room containing a player or virtual character who is sick) may have contributed to the feeling of embodiment.

\section{Understanding of the Game as a Dynamic System}

Our second research question asks if students understand the dynamic nature of the model underlying the game. As shown by their interview responses and influence diagrams, some students did understand the game as a complex dynamic system. They understood that their actions affected the way events unfolded in the game, such as who got sick and when. They appreciated the exponential growth of disease spread, and the potentially large consequences of a small event such as a single infection. They also showed evidence that some students understood the complex causality of the game, in which different factors are interconnected by many causal links.

This understanding of the game as a dynamic system may have been a crucial element of the authenticity of the experience described above. The threat of infection was made more meaningful to students by the awareness that their own actions and those of the whole team had a direct impact on it. The special capabilities of each role become much more important in a dynamic game world, because they contribute to the inquiry processes made possible in participatory simulations.

\section{Misconceptions About Disease Transmission}

While students did grasp the dynamic nature of the model in the game, they may have misunderstood some details of the disease transmission mechanism. Specifically, they seemed to view the presence of sick people as 
having a continuously detrimental effect on their own health, rather than exposing them to the possibility of a discrete infection event. They also may have had a related misconception about the function of vaccines. Possibly, students draw this mental model of disease from everyday experience and intuition, and the game did not provide enough feedback to challenge it; in fact, events in the game can easily be construed as consistent with it. It is also possible that the game generated these misconceptions. The simple description of disease transmission given to students in the pre-game briefing may have been insufficient to distinguish it from their existing ideas. Also, the invisibility in the game of the underlying mechanism makes it difficult to make inferences about it. Players have only indirect access to information about another player's underlying disease state (an antigen count). For example, an infected player's health meter begins to drop only after a time delay has elapsed, and the antigen count in the body can only be viewed by taking a sample and analyzing it.

It may be possible to improve students' understanding of disease transmission in the game by making a more detailed and explicit presentation at the beginning of the game. This might use visualizations of virus particles replicating inside a person's body, then leaping to another person and replicating there. Another possibility would be to provide a more direct and instantaneous way of viewing antigen counts in the game. One drawback is that this would weaken some of the game mechanics that reward sophisticated strategies (such as time delays that require planning and prediction, and limited access to information, which requires inferences that combine many sources). On the other hand it would expose the underlying model to more direct observation, and perhaps encourage students to carry out more explicit experimentation.

Another way to improve understanding in the game would be to include assistance as described by Dede et al. (2005a) to support inquiry in a multi-user virtual environment. In that case, experts modeled inquiry for students, and provided coaching. The experts were avatars embedded directly in the virtual environment. In Outbreak @ The Institute, live facilitators could potentially play the role of experts. Also, the functionality of virtual characters could be enhanced to detect certain conditions in the game and provide contextually relevant suggestions. For example, an assistant character could appear when players have just encountered an infected virtual character for the first time and suggest they try a diagnostic test, describe the likelihood at that time of becoming infected and point out the various preventative measures available.

\section{Future Directions}

Outbreak@ the Institute is the first example of a "participatory reality" game, incorporating the affordances of both the location basis of augmented reality games and the dynamic models of participatory simulations. Together these affordances make possible new kinds of authentic science inquiry experiences. Future games of this type could benefit from advances in wireless technology, such as positioning techniques that would make it possible for the game to locate players with high resolution, indoors and outdoors, while still allowing fast communication to a server. The dynamic models underlying the game could be extended to include a diverse range of phenomena, from microeconomic activity to climate change. The virtual characters could include a wider range of dynamic states, making them more life-like and interactive. Embedded experts could support better student understanding of underlying world models. A technological infrastructure for performance assessment within the game could be a powerful tool for evaluating student performance in authentic problem solving. These improvements would expand the range and depth of possible future games, which could cover a wide range of content areas in science, engineering and the humanities.

\section{REFERENCES}

Barab, S. A., and Duffy, T. (2000). From practice fields to communities of practice. In D. Jonassen, \& S. Land (Eds. Theoretical Foundations of Learning Environments Hillsdale, NJ: Lawrence Erlbaum Associates.

Bereiter, C. (2002). Education and Mind in the Knowledge Age, Lawrence Erlbaum Associates, Publishers, Mahwah, New Jersey.

Colella, V. (2000). Participatory simulations: building collaborative understanding through immersive dynamic modeling. Journal of the Learning Sciences 9(4): 471-500.

Dede, C., Ketelhut, D. J., Clarke, J., Nelson, B., and Bowman, C. (2005a). Students' motivation and learning of science in a multi-user virtual environment. Paper presented at the American Educational Research Association Conference, Montreal, Canada.

Dede, C., Korte, S., Nelson, R., Valdez, G., and Ward, D. (2005b). Transforming Education for the 21st Century: An Economic Imperative, Learning Point Associates, Chicago, IL.

Dewey, J. (1938). Experience and Education. Simon \& Schuster.

Klopfer, E., and Squire, K. (2006). Environmental detectives - the development of an augmented reality platform for environmental simulations. Educational Technology Research and Development (in press). 
Klopfer, E., Yoon, S., and Rivas, L. (2004). Comparative analysis of palm and wearable computers for participatory simulations. Journal of Computer Assisted Learning 20: 347-359.

Klopfer, E., Yoon, S., and Perry, J. (2005). Using palm technology in Participatory Simulations of complex systems: a new take on ubiquitous and accessible mobile computing. Journal of Science Education and Technology 14(3): 285-298.

Lave, J., and Wenger, E. (1991). Situated Learning: Legitimate Peripheral Participation, Cambridge University Press, New York.

National Science Teachers Association. (2003). Standards for Science Teacher Preparation, National Science Teachers Association, Arlington VA.
Roschelle, J. M., Pea, R. D., Hoadley, C. M., Gordin, D. N., and Means, B. M. (2000). Changing how and what children learn in school with computer-based technologies. Children and Computer Technology 10(2): 76-101.

Senge, P. (2000). Schools That Learn, Doubleday, New York, NY. Squire, K., and Klopfer, E. (2006). Case study analysis of augmented reality simulations on handheld computers. The Journal of the Learning Sciences in review (revised and resubmitted)

Wilensky, U., and Stroup, W. (1999). Learning through participatory simulations: network-based design for systems learning in classrooms. Computer Supported Collaborative Learning (CSCL'99). Stanford University: December 12-15, 1999. 